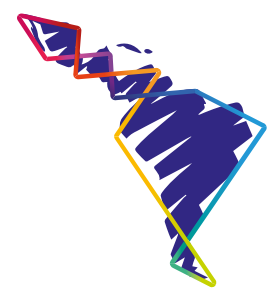

\title{
Marco conceptual y enfoques en la promoción de la salud en Costa Rica
}

\section{Conceptual framework and approaches for health promotion in Costa Rica}

\author{
Lidia Isabel Picado Herrera' \\ Raúl Fonseca Hernández²
}

\begin{abstract}
Resumen
El artículo tiene como objetivo realizar un análisis teórico respecto a las bases conceptuales y a los enfoques que se han construido alrededor de la promoción de la salud (PS), con el propósito de identificar las similitudes, divergencias, avances y oportunidades para fortalecer la posición teórica coherente, con la filosofía de promoción de salud, desde una visión positiva, propositiva, proactiva e innovadora que se impulsa desde Costa Rica. Metodológicamente se utiliza el análisis de contenido, dado el enfoque eminentemente teórico del artículo. El objeto de estudio es el desarrollo conceptual y teórico de la PS y algunas de sus visiones. Se discute sobre algunas corrientes teóricas vinculadas al tema, entre ellas, la definición impulsada por OPS, en la Carta de Otawa 1986, estilos de vida saludables, y la capacidad de potenciar el desarrollo humano para luego enfatizar en la visión positiva que se fomenta desde el Modelo de Rectoría de Promoción de la Salud del Ministerio de Salud, Costa Rica; se hace alusión a diferencias entre PS y prevención en general, incluidas las relacionadas con derechos y necesidades. Se concluye con la trascendencia que tiene el tema en cuestión, sus aportes y su relevancia para mejorar la implementación del modelo de PS en la región de Occidente, en primera instancia, pudiendo extenderse a otras regiones o incluso países.
\end{abstract}

Palabras clave: promoción de la salud (PS); reconceptualizaciones; modelo de rectoría; factores protectores.

\section{Summary}

The purpose of the article is to carry out a theoretical analysis regarding the conceptual bases and the approaches that have been built around Health Promotion (HP), with the purpose of

1 Doctora. Trabajadora Social Salubrista e investigadora. Ministerio de Salud.

2 Máster. Economista, académico e investigador, de la Sección de Economía de la Sede de Occidente de la Universidad de Costa Rica y de la Escuela de Relaciones Internacionales de la Universidad Nacional. 
identifying similarities, divergences, advances and opportunities to strengthen the coherent theoretical position with the philosophy of Health Promotion, from a positive, purposeful, proactive and innovative vision promoted from within Costa Rica. Methodologically, content analysis is used, given the eminently theoretical approach of the article. The object of study is the conceptual and theoretical development of HP and some of its visions. The article discusses some theoretical currents linked to the topic, including the definition forwarded by PAHO in its 1986 Otawa Charter, healthy lifestyles, and the ability to enhance human development, to then emphasize the positive vision that is promoted by the Health Promotion Rectory Model of the Ministry of Health of Costa Rica; reference is made to differences existing between HP and prevention in general, including those related to rights and needs. The work concludes with the importance of the subject in question, its contributions and relevance to improve the implementation of the HP model in the Región de Occidente of Costa Rica (Costa Rica's Western Region), in the first instance, with possible dissemination to other regions or even other countries.

Keywords: Health Promotion (HP); Reconceptualizations; Rectory model; Protective factors.

\section{Introducción}

La salud es un derecho humano universal. En su análisis intervienen diferentes enfoques que pretenden orientar, así como facilitar el acceso de toda la población y, por ende, contribuir al mejoramiento de la calidad de vida de esta. Al igual que la salud, la promoción de la salud históricamente ha sido interpretada desde diversos enfoques, por consiguiente, los métodos y relevancia que se le atribuyen en su abordaje han variado al responder a la realidad social cambiante, según características territoriales, políticas y económicas de cada época.

La PS es catalogada como una función esencial de Salud pública, sus actuaciones están vinculadas al análisis de los derechos humanos, las cartas internacionales e iniciativas gestadas a nivel mundial; no obstante, su orientación se enmarca en una lógica donde prevalecen las acciones preventivas, por consiguiente, dificulta su diferenciación con el enfoque de prevención de enfermedades.

En este sentido, se detecta falta de claridad conceptual, así como metodológica que limita la diferenciación y operativización entre promoción de la salud y prevención de enfermedades. Esta situación conlleva a que se privilegien las acciones y presupuesto para el segundo enfoque, en detrimento del avance del primero, contrario al discurso de diversas instancias internacionales y nacionales de darle prioridad. Debido a ello, se confirma la necesidad de ahondar en aspectos teóricos que caractericen y consoliden la PS desde una perspectiva que marque diferencia en su definición, funcionalidad, así como en los resultados esperados. 
En el marco del proyecto titulado "Evaluación de resultados de la aplicación del enfoque positivo de PS en la Región Occidente", que desarrolla la Sección de Economía del Departamento de Ciencias Sociales de la Sede de Occidente, de la Universidad de Costa Rica en conjunto con la coordinadora regional de PS, de la Unidad de Rectoría de la Región de Salud Occidente del Ministerio de Salud, surge este artículo como un producto de dicho proyecto. Así mismo, el análisis de la salud, desde la visión de la promoción, es una variable social que explica la competitividad territorial de los cantones periféricos, por tanto, aporta también elementos claves vinculados al proyecto de la Universidad Nacional titulado "Análisis de variables socioeconómicos que explican la competitividad territorial y el potencial exportador de las regiones periféricas en Costa Rica".

El objeto de estudio es el desarrollo conceptual y teórico de la PS, así como realizar un análisis teórico respecto a las bases conceptuales y a los enfoques que se han construido alrededor de la PS. Metodológicamente se trabaja el contenido, para lo cual se usan las siguientes categorías de análisis: Definiciones y enfoques de PS con énfasis en el enfoque positivo, proactivo, propositivo e innovador del Modelo de Rectoría en PS, Ministerio de Salud de Costa Rica (MS) que guía la investigación señalada en párrafos precedentes.

\section{La PS desde la perspectiva de la carta de Otawa y sus líneas de acción}

La Carta de Ottawa de 1986 (Organización Mundial de la Salud [OMS], 1986), indica que la PS constituye una estrategia global, política y social que abarca, no solamente las acciones orientadas a fortalecer las habilidades y capacidades de los individuos, sino también las dirigidas a robustecer las condiciones sociales, ambientales y económicas requeridas para impactar positivamente en la salud colectiva e individual.

De igual forma, la (OMS, 1986) plantea el propósito de la PS como:

...proporcionar a los pueblos los medios necesarios para mejorar su salud y ejercer un mayor control sobre la misma, en este sentido trasciende la idea de formas de vidas sanas para incluir las condiciones y requisitos para la salud que son: paz, vivienda, educación, alimentación, renta, ecosistema estable, recursos sostenibles, justicia social y equidad. (p. 2)

El enfoque en mención establece cinco líneas de acción: generación de políticas públicas saludables, creación de ambientes saludables, fortalecimiento de la participación social, desarrollo de potencialidades individuales y colectivas, y 
reorientación de servicios de salud. Entre los componentes y estrategias importantes para aplicar en su implementación destacan: educación para la salud (formal e informal), comunicación social, la intersectorialidad, las alianzas estratégicas, las redes de apoyo social, la abogacía de la salud, el mercado, la planificación, la investigación.

En este marco, las líneas de acción adquieren relevancia por cuanto constituyen herramientas y estrategias para orientar y facilitar las acciones de PS; de esta manera, las políticas públicas representan las leyes, decretos reglamento y normas, por consiguiente, son los referentes legales y técnicos que permiten el abordaje de la situación de salud de la población; estas mismas se deben construir desde procesos de participación social con representación de todos los sectores públicos y organizaciones comunales, entre otros, en procura de dotar de conocimientos, favorecer el desarrollo de habilidades y potencialidades individuales y colectivas mediante el empoderamiento, fomento de capacidades e información para la toma de decisiones que facilite el control sobre la vida, condiciones para una vida sana y el acceso equitativo a bienes y servicios; todo ello para reforzar la práctica de comportamientos saludables y de habilidades que propicien proyectos de vida con sentido positivo, de igual manera, que ayuden a resistir las presiones del medio. Por otra parte, la línea de creación de ambiente saludables se enfoca en todos aquellos aspectos favorables del entorno: físico, social, emocional, económico, ecológico y político saludable que influencian la salud. Así mismo, la correspondiente a reorientación de los servicios de salud implica adaptar las estructuras y funciones a las nuevas demandas de salud, es decir, priorizar la actuación de los servicios sobre los determinantes y sus factores protectores de la salud (conciencia y conducta, económico-ecológico, biológicos). Requiere de alianzas con otros sectores para influenciar en la atención integral de factores protectores que condicionan la salud.

Aunque se reconocen las buenas intenciones y planteamientos teóricos establecidos en la Carta de Otawa, entre ellas las líneas de acción mencionadas, en la práctica, la visión prevalente es preventiva, pues apunta al abordaje de los factores de riesgo, dejando de lado la planificación y, por consiguiente, las actuaciones sobre aquellas condiciones, situaciones favorables que deben ser también privilegiadas para presérvalas y fortalecerlas.

\section{Enfoques teóricos que se han construido alrededor del tema de la PS}

Si bien el concepto de PS aportado en la Carta de Ottawa es predominante a nivel internacional, no sucede lo mismo sobre el enfoque con el que se aborda 
este mismo. Se han identificado diferencias en cuanto a la forma de visualizar su implementación o abordaje; para efectos de este artículo, se mencionan los enunciados por Carmona, Rozo y Mogollón (2005), así como Eslava-Castaneda (2002), dada su complementariedad con la visión positiva, propositiva, proactiva e innovadora de PS del Ministerio de Salud, Costa Rica, que se desarrollará posteriormente.

Según Carmona, Rozo y Mogollón (2005), en la actualidad es posible identificar dos enfoques teóricos y metodológicos que explican qué significa promover la salud. El primero, denominado "estilos de vida saludables" se vincula con las condiciones o formas de vida, actitudes o comportamientos del ser humano, de igual forma con el autocuidado, por lo que la responsabilidad de promover estos estilos de vida queda en manos de las instituciones prestadoras de los servicios en salud.

A pesar de que este enfoque permitió trascender la manera como se prestan los servicios de salud en una perspectiva médico-asistencialista, sus acciones continuaron dándose desde lo preventivo y manifestaron, en el plano individual del ser humano y no en la parte colectiva de la sociedad. Otra crítica es que se concentra únicamente en actividades recreativas, deportivas y de alimentación saludable, y resulta insuficiente y muy reduccionista en relación con los determinantes y factores protectores que forman parte de las condiciones requeridas para mantener la salud, la calidad de vida y el desarrollo humano, como se explica a continuación. El campo de los estilos de vida saludables es, realmente, más amplio, implica trabajar con las condiciones o formas de vida, actitudes o comportamientos del ser humano.

Desde una perspectiva integral, es necesario considerar los estilos de vida como parte de una dimensión colectiva y social, que comprende tres aspectos interrelacionados: el material, el social y el ideológico (Bibeau \& col, 1985, citado en Picado, 2017b). Señala este autor que, en lo material, el estilo de vida se caracteriza por manifestaciones de la cultura material: vivienda, alimentación, vestido; en lo social, según las formas y estructuras organizativas: tipo de familia, grupos de parentesco, redes sociales de apoyo y sistemas de soporte como las instituciones y asociaciones; en el plano ideológico, los estilos de vida se expresan a través de las ideas, valores y creencias que determinan las respuestas o comportamientos a los distintos sucesos de la vida.

Según Maya (2001), los estilos de vida saludables deben estar concatenados a los diversos contextos en los que se desenvuelve la persona, tales como económicos, políticos, culturales y sociales. En este sentido, debe imprimirse un enfoque integral, 
alejado de una perspectiva aislada. El objetivo fundamental de los estilos de vida saludables es mejorar las condiciones de vida y el bienestar general de las personas.

En este marco, el desarrollo de la estrategia para generar estilos de vida saludables pasa por propiciar y potenciar un compromiso tanto a nivel individual como social. Esta responsabilidad debe generar acciones colectivas que, con el pasar del tiempo, integren elementos culturales formentadores de hábitos que modifiquen conductas de las personas y la sociedad en general, para poder, así, mejorar el bienestar y la calidad de vida de quienes incorporan estos comportamientos positivos en su diario vivir. $\mathrm{El}$ iniciar este proceso puede ser complicado, pero debe atenderse a la mayor brevedad posible (Cáez y Casas, 2007).

El segundo enfoque señalado por Carmona et al. (2005) es denominado como la capacidad de potenciar el desarrollo humano (DH). Se considera que $\mathrm{DH}$ es un proceso de transformación que persigue el mejoramiento de las condiciones para lograr una mejor calidad de vida y alcanzar a mantener la dignidad de la persona humana en la sociedad. Esta transformación exige la participación de la población.

El objetivo final es humanizar y dignificar al ser humano a través de la satisfacción de sus necesidades de expresión, creatividad, participación, igualdad de condiciones de convivencia y autodeterminación, vinculado a las oportunidades de autorrealización de las personas de manera individual y colectiva. Por lo tanto, el enfoque busca "promover la responsabilidad social de la salud, incrementar la inversión para el desarrollo de la salud, consolidar y expandir nuevas alianzas para la salud, aumentar la capacidad de la comunidad, crear poder de los individuos (empoderamiento social)" (Picado, 2014, p. 154), así como mantener y aumentar la infraestructura que se dispone en materia de PS. En razón de ello, Carmona et al. (2005) consideran la PS como un conjunto de acciones, con el fin de que el bienestar se convierta en la tarea social más importante, no solo del individuo, sino de la sociedad.

Es así como del DH trasciende el sano desarrollo biológico del individuo, para penetrar en el campo de lo social, lo económico, lo cultural y debe procurar calidad de vida.

Por otra parte, Eslava-Castaneda (2006) genera un enfoque innovador en la PS, en el cual se identifican las acciones que se pueden hacer (¿qué hacer?) y aplicar en los diferentes programas, actividades y proyectos. En este sentido, se propone sustituir el enfoque del problema en la salud, o el prevenir la enfermedad, por el precepto que busque el alcanzar objetivos de satisfacción, en los cuales se logren 
identificar las condiciones favorables para alcanzar dichos objetivos positivos, los cuales deben perseguirse siempre, aún en ausencia de enfermedad, con la idea de propiciar un estilo de vida saludable.

Como se observa, existe coincidencia entre en el enfoque de la capacidad de potencializar el DH con el siguiente enfoque planteado por Eslava-Castaneda (2006), en cuanto a la relevancia del empoderamiento de las personas y las comunidades, a fin de potencializar lo positivo y ejercer control sobre los determinantes de la salud.

Estos planteamientos, como se mencionó, guardan congruencia con los del Ministerio de Salud, según se observa a continuación.

\section{Enfoque en PS desde una visión positiva, proactiva, propositiva e innovadora}

El presente apartado presentará la reconceptualización, estrategias y herramientas de la PS desde la visión e instrumentalización que ha realizado en el tema el Ministerio de Salud de Costa Rica. Posteriormente, se analizará el entorno vinculado al escenario de salud, para, finalmente, definir el concepto de factores protectores.

\section{Reconceptualizaciones, estrategias y herramientas desde la perspectiva del Ministerio de Salud de Costa Rica}

Costa Rica es signatario de compromisos internacionales definidos en torno a PS, de igual forma cuenta a nivel nacional con un marco jurídico vigente, planes, programas y proyectos nacionales que contienen explícita o implícitamente competencias en este campo, por lo tanto, se determinan compromisos de los actores sociales de instancias públicas y otros sectores sociales, en la construcción de la salud en general y del enfoque positivo de PS en particular

En este sentido, el Ministerio de Salud, ente rector de la salud en Costa Rica, cuenta con el modelo de Rectoría en PS (Ministerio de Salud, 2017), el cual es una representación teórica, que reconceptualiza términos básicos, define las funciones gerenciales rectoras generales y específicas, las herramientas, las estrategias que sustentan su quehacer de acuerdo con el marco legal internacional, nacional, y técnico-político y estratégico institucional vigente.

Dicha rectoría tiene el reto de asumirla desde la perspectiva de gerencia social pública (Picado, 2017b), dado que su gestión trasciende de lo institucional a los 
ámbitos sectoriales e intersectoriales; por tal razón, está obligada a reconocer y fortalecer sus habilidades, conocimientos, manejo de herramientas. En fin, ajustar y aplicar todos los aspectos y principios inherentes, con las personas representantes de esos ámbitos, en busca de generar espacios de trabajo armoniosos, efectivos, motivadores e inspiradores y de compromiso, en especial con quienes conforman grupos o equipos de trabajo en las diversas modalidades de actuación como comisiones, redes, entre otras para el desarrollo articulado de los procesos de PS.

Este apartado contiene las reconceptualizaciones, estrategias y herramientas planteadas en el Modelo de Rectoría en PS, como base para conducir los procesos y acciones desde la función rectora de PS, que favorezcan el abordaje de los determinantes y factores protectores de la salud como derecho esencial de las personas y colectividades; de igual forma, por ser condiciones fundamentales para la calidad de vida, esos componentes constituyen referentes de la investigación que motiva este artículo. De igual forma, se considera pertinente aducir sobre las diferencias entre promoción y prevención de enfermedades, por considerar este punto relevante, ante la falta de claridad predominante en actores sociales con responsabilidad en este campo.

Entre las funciones rectoras del Ministerio de Salud, se encuentra la correspondiente a PS, definida de la siguiente manera:

Función sustantiva responsable de liderar o articular, de manera efectiva, los esfuerzos y recursos de los actores sociales en la construcción de políticas y normativas, el análisis de situación, la planificación, la evaluación e investigación y verifica el posicionamiento de la PS y su enfoque positivo en los procesos de formación y capacitación del recurso humano, en el ámbito nacional en el escenario de salud, orientados a potencializar y desarrollar los factores protectores, determinantes y asociados, que generan y fortalecen desde una visión positiva, la salud en la población. (Ministerio de Salud, 2017, p. 20)

Para cumplir con la rectoría técnica en PS, se establecieron las siguientes funciones:

\section{Cuadro 1: Funciones generales de rectoría en PS}

Direccionar esfuerzos y recursos de los actores sociales hacia el desarrollo de los factores protectores, determinantes y condicionantes, que generan y fortalecen la salud en la población.
Conducir y articular con actores sociales para el análisis de factores protectores, proceso orientado a identificar y establecer la manera en que generan y fortalecen la salud de la población. 
Conducir el componente de PS en los procesos de planificación con actores sociales
Conducir el desarrollo del componente de PS en las políticas, planes, programas y proyectos relacionados con salud desde su visión positiva

Conducir y verificar el posicionamiento del Coordinar y promover el desarrollo de enfoque positivo de la PS en los procesos investigaciones en promoción de la salud de formación y capacitación del recurso humano, en el ámbito nacional.

Fuente: Ministerio de Salud, 2017.

Acorde con la revisión bibliográfica realizada y la experiencia institucional acumulada, el Equipo Técnico Asesor de Rectoría en PS ha elaborado la siguiente definición para PS:

La PS constituye un proceso integral, transformador y dinámico, de análisis, potencialización y de trabajo articulado con actores sociales para el abordaje de los factores protectores, determinantes y condicionantes, que generan y fortalecen desde una visión positiva, la salud en la población. (Ministerio de Salud, 2017, p. 18)

PS se fomenta desde el enfoque positivo, proactivo, propositivo e innovador; orienta sus actuaciones desde la salud, por la salud y para la salud, pues parte de la identificación de determinantes y factores protectores de esta; formula objetivos positivos; genera propuestas de acción innovadoras, proactivas para abordarles. En este sentido, aprovecha las condiciones favorables y las oportunidades que contribuyen a la protección y fortalecimiento de la salud. Dicho enfoque integra otros actores y acciones que están fuera del Sector salud, pues la PS debe garantizarse tanto desde las acciones generadas por el Estado (que evidentemente debe tener un papel protagónico), así como desde diversas organizaciones privadas y civiles que apoyen las diversas estrategias de PS. El objetivo último es que se logren propiciar iniciativas que apoyen la PS desde diferentes ámbitos, tales como legales, políticos, económicos, de recreación, ambientales, los cuales logren permear las diferentes poblaciones (niñez, juventud, adultez, personas adultas mayores) con el objetivo de mejorar el bienestar individual y colectivo (Picado y Quesada, 2014).

A continuación, se razona sobre la PS con enfoque positivo, proactivo, propositivo e innovador: La promoción de la salud parte del enfoque positivo que consiste en planificar y actuar, a partir de lo que está bien, potenciando y desarrollando procesos vistos desde las posibilidades, fortalezas y oportunidades en contraposición a las carencias. Propositivo, pues ante las diversas realidades, características culturales, económicas y políticas, exige la revisión y diseño de propuestas, 
alternativas o iniciativas que contribuyan a la toma de decisiones en cuanto al posicionamiento de nuevo paradigma de salud. Además, procura la generación de la cultura de salud desde la lógica positiva que se ajuste a los constantes cambios en una perspectiva proactiva, a fin de anticiparse en el desarrollo de procesos necesarios con iniciativa, emprendedurismo y perseverancia.

La innovación social también es fundamental ante lo cambios señalados. Amerita nuevas ideas, productos, servicios y modelos de relaciones sociales que motiven a la sociedad a ser parte activa en la construcción social, implementación de alternativas y condiciones para su calidad de vida, su bienestar y desarrollo.

Interesa resaltar, en cuanto al carácter innovador del enfoque de PS, la incorporación de nuevas técnicas, entre ellas, la programación neurolingüística (PNL) con enfoque participativo, en procura de lograr mayor efectividad de las estrategias, metodologías de capacitación y planificación con actores sociales para facilitar su ejecución. Sus herramientas básicas permiten proporcionar una modificación de los mapas mentales y perceptuales de las personas para que valoren y propongan paradigmas nuevos que permitan el progreso, su desarrollo; mejorar sus canales y formas de comunicación, sus procesos de enseñanza-aprendizaje; diseñar estrategias dirigidas a establecer criterios de funcionabilidad en la evaluación del impacto de las acciones.

Entre las formas de ver el cambio en PNL se encuentra el generativo, el cual propone un curso de acción destinado a mejorar lo que está funcionando bien para mantenerlo así y, en el mejor de los casos, aumentar ese bienestar. El interés es fomentar y generar las condiciones apropiadas en niveles personales, sociales, económicos, ambientales y estatales, planteamientos congruentes con la lógica positiva de PS.

También se acuña la comunicación para el desarrollo, la cual busca darle mayor contenido a la comunicación, ubicándola en los procesos culturales, sociales y políticos, y recuperando su sentido educativo-participativo, apostando por una comunicación ciudadana vinculada a gestar procesos de desarrollo que se articulen a la vida cotidiana de la gente y que orienten la acción ciudadana hacia el cambio social.

\section{Promoción de la salud y su contribución al desarrollo humano}

Los diferentes actores sociales deben participar en las diversas etapas del proceso de diseño e implementación de la PS, tanto a nivel nacional como local. Desde los procesos introductores de capacitación, pasando por las diferentes etapas de formulación, hasta la aplicación y posterior evaluación de los resultados y del impacto generado, deben estar presentes tanto los actores estatales, como los 
privados, así como los colectivos sociales. De tal forma que se actúe sobre los determinantes sociales y sus factores protectores, en procura del fortalecimiento de las condiciones, y comportamientos saludables, así como de la calidad de vida en la población.

Mediante sus planes, programas, proyectos y sus procesos de acción, se estimulan sistemas, vinculaciones o interacciones de relaciones humanas, aplicando métodos, estrategias, herramientas e instrumentos que innovan la forma de trabajar con los actores sociales en procura de fomentar el cambio en el paradigma, mediante el cual se construya colectivamente una verdadera cultura de salud, cuyos resultados apunten al desarrollo social y humano. Esta lógica del enfoque en mención permite:

Integrar actuaciones que buscan generar políticas públicas vinculantes al desarrollo humano para impactar favorablemente en las condiciones, calidad de vida y sus determinantes, desde el escenario de salud. Para ello, debe generarse un desarrollo sistemático en los procesos y actividades diarias, de tal forma que las personas que participan en diferentes organizaciones pueden conocer las herramientas y técnicas necesarias que permitan saber sobre el enfoque positivo y lo incorporen como una capacidad y habilidad en su cotidianidad. Esto generará, en el mediano y largo plazo, condiciones de vida saludables para estos individuos y para la sociedad en general. Para ello, es necesario abrir y propiciar espacios locales, regionales y nacionales, para planificar acciones colectivas que involucren a personas e instituciones en la ejecución de acciones priorizadas por el enfoque de PS (Picado, 2017a).

Es importante recordar que al ser el cambio la constante, las organizaciones institucionales públicas y otras vinculadas al tema en cuestión deben también establecer momentos de revisión e investigación que permitan realizar oportunamente los ajustes y actualizaciones teórico-conceptuales, normativas, metodológicas y operativas que se requieran para que la promoción de la salud logre resultados efectivos e impacte en las condiciones, en el desarrollo humano y del país en general.

A continuación, se definen otros conceptos ligados a los principios estratégicos que conlleva la implementación de la PS, visón positiva objeto de este artículo.

\section{Análisis de entorno vinculado al escenario de salud}

En esta caracterización del contexto se destacan los factores protectores determinantes y condicionantes de la salud, procesos sociales y sus vinculaciones; se desarrollarán las políticas, programas y proyectos sociales; se realiza un análisis y acciones proyectivas frente a los cambios que se introducen mediante acciones 
innovadoras. El gran desafío de los gerentes sociales responsables de conducir a los actores sociales hacia la construcción de la salud consiste en evaluar la incertidumbre del entorno y proponer estrategias seguras en medio de tales condiciones para garantizar la efectividad de las actuaciones e impacto en salud (Picado, 2017a).

Respecto de la calidad de vida, Rodado y Grijalba (citados en Teodoro et al., 2003) la definen como las "condiciones y circunstancias en que se desenvuelve la existencia de una persona o de un grupo social en un ambiente determinado" (p. 11), indican que está estructurada por cuatro componentes estrechamente conectados que deben surgir complementariamente para que la dignidad de la existencia sea realidad. Entre esas dimensiones destaca las condiciones de vida:

Conjunto de bienes que conforman la parte social de la existencia humana, o sea, la oferta empresarial e institucional de servicios en el campo de la seguridad social, la recreación, la cultura, la educación, la vivienda, entre otros, además de aspectos como libertades públicas, respeto a los derechos humanos, espacios de participación social y vida espiritual, todo lo cual hace posible el desarrollo integral de las personas.

Otros componentes y conceptos relevantes para las actuaciones de PS, cuyas definiciones fueron construidas a partir de la experiencia de profesionales del Ministerio de Salud, Dirección Regional de Rectoría de la Salud Central de Occidente en concordancia con requerimientos del enfoque positivo planteado, son los siguientes:

1. Escenario de salud: Este primer componente constituye el campo de análisis de las situaciones de salud, desde su esencia misma, puesto que pretende generar y fortalecer la salud de la población, a partir de un especial énfasis a la calidad de vida positiva, y del establecimiento y desarrollo de factores y determinantes que se encuentran en relación directa con la salud. Cabe rescatar que el escenario de salud tiene como función la planificación de las actuaciones de actores sociales como el Ministerio de Salud, en su función rectora, dentro del proceso de PS (Cabrera \& Picado, citados en Picado, 2017).

2. Experiencias exitosas saludables: Es el proceso o conjunto de actividades significativas que tienen como propósito fortalecer las condiciones y calidad de vida de las personas, lo cual beneficia al desarrollo humano individual y colectivo de quienes residen en un espacio geográfico determinado. Como parte de este segundo punto, es menester señalar que las actividades significativas se llevan a cabo por periodos mayores a un año, y son ejecutadas por una o varias instituciones en conjunto, una persona o grupos de personas pertenecientes a comunidades, o bien, por grupos con una organización formal o informal (Picado, 2017). 
3. Experiencia en PS: Este componente implica directamente diversos ámbitos de la sociedad, tales como aspectos ambientales, espirituales, de salud, políticos, socioculturales, ambientales, económicos, entre otros; con el propósito de maximizar las condiciones y calidad de vida que favorece el desarrollo personal y social de la ciudadanía de un país. Dicho propósito se logra mediante la implementación de planes o acciones continuas durante periodos de al menos un año o más; estas mismas son ejecutadas tanto por entes estatales, privados o civiles (Picado, 2017).

4. Factor protector: Son las condiciones positivas (variables) biológicas, políticas, ambientales, sociales, económicas y espirituales, que favorecen la salud y, por consiguiente, la vida humana, individual y colectiva de los ecosistemas, coadyuva a que se den procesos beneficiosos que propician un desarrollo integral (Cabrera y Picado, citados en Picado, 2017).

A continuación, se presentan las principales herramientas y estrategias para gestionar procesos de PS:

La labor con actores sociales representa la clave para la formulación e implementación de las políticas, planes, programas, proyectos y actuaciones sociales, de ahí la importancia de la aplicación de estrategias que permitan fortalecer la gestión de los procesos de promoción de la salud, entre ellas destacan la abogacía de la salud y alianzas estratégicas: multisectorialidad, participación social en salud, redes de actores sociales, elaboración y firma de cartas de entendimiento o compromisos, negociación y concertación, análisis conjunto con actores sociales de normativa y competencias institucionales, análisis permanente de contexto Institucional, nacional e internacional, caracterización y análisis de actores sociales.

En el caso de herramientas resaltan: investigación, estudios de línea base, planificación, evaluación, sistemas de información, mapeo y georreferenciación de actores sociales y proyectos vinculados a PS; también son importantes las herramientas comunicacionales tales como: programación neurolingüística con enfoque participativo, enfoques -técnicas educativas- medios de comunicación social, enfoques y nuevas técnicas educativas, comunicacionales: presenciales, virtuales, mixtas, teleconferencias, redes sociales, entre otras.

\section{Diferencias entre PS y prevención de enfermedades}

Una vez analizados los conceptos, estrategias, herramientas y componentes anteriores, el objetivo principal es resaltar y facilitar como una guía para interpretar y orientar los procesos de PS desde su enfoque positivo, propositivo, proactivo e 
innovador. Se considera importante comprender la lógica de actuación destacando las diferencias más relevantes entre ambos enfoques, por cuanto a la fecha se observa, en diversos actores sociales con responsabilidad en su implementación, falta de claridad entre ambos y, por consiguiente, se continúa confundiendo y privilegiando la prevención. En el cuadro 2 siguiente se destacan las diferencias, las cuales, a su vez, se constituyen en características de cada uno.

\section{Cuadro 2: Diferencias entre PS y prevención de enfermedades}

\begin{tabular}{|c|c|}
\hline PS: Características & $\begin{array}{l}\text { Prevención de la enfermedad: } \\
\text { Características }\end{array}$ \\
\hline Visión salutogénica & Visión patogénica \\
\hline Modelo social & Modelo biomédico - asistencial \\
\hline $\begin{array}{l}\text { Actuación sobre determinantes y factores } \\
\text { protectores de la salud, implica mantener } \\
\text { procesos para generar cambios. }\end{array}$ & $\begin{array}{l}\text { Actuación sobre factores de riesgo, acciones } \\
\text { puntuales }\end{array}$ \\
\hline $\begin{array}{l}\text { Representa una nueva lógica de trabajo, } \\
\text { pues prioriza y planifica desde para y por lo } \\
\text { positivo. }\end{array}$ & $\begin{array}{l}\text { Prioriza y planifica para actuar y mitigar } \\
\text { factores de riesgo }\end{array}$ \\
\hline $\begin{array}{l}\text { Aplicación de métodos, técnicas y } \\
\text { estrategias para favorecer las condiciones } \\
\text { salutogénicas }\end{array}$ & $\begin{array}{l}\text { Aplicación de técnicas para reducir los } \\
\text { factores de riesgo y enfermedad o daño }\end{array}$ \\
\hline $\begin{array}{l}\text { Busca fortalecer las condiciones y calidad } \\
\text { de vida desde lo favorable (positivo). } \\
\text { Escenario de salud }\end{array}$ & $\begin{array}{l}\text { Busca atenuar los riesgos para disminuir } \\
\text { complicaciones de enfermedad }\end{array}$ \\
\hline $\begin{array}{l}\text { Se dirige a población en general como } \\
\text { comunidades o grupos específicos }\end{array}$ & $\begin{array}{l}\text { Se dirige a personas y grupos de riesgo, } \\
\text { cobertura limitada. }\end{array}$ \\
\hline $\begin{array}{l}\text { Busca desarrollar potencialidades positivas, } \\
\text { fortalezas individuales y colectivas }\end{array}$ & $\begin{array}{l}\text { Se limita a la aplicación de técnicas para } \\
\text { evitar la aparición, o avance de una } \\
\text { enfermedad o daño }\end{array}$ \\
\hline Enfoque de derechos & Privilegia solo grupos de riesgo \\
\hline Es proactivo, propositivo. Inclusivo & Es reactiva. Excluyente \\
\hline $\begin{array}{l}\text { Fortalecimiento de procesos de } \\
\text { participación social intersectorial }\end{array}$ & $\begin{array}{l}\text { Débil participación en escenarios políticos } \\
\text { e intersectoriales }\end{array}$ \\
\hline $\begin{array}{l}\text { Acciones educación e información en salud } \\
\text { hacia el reforzamiento de estilos saludables }\end{array}$ & $\begin{array}{l}\text { Acciones de educación e información } \\
\text { hacia la protección de los individuos de los } \\
\text { factores de riesgo o problemas. }\end{array}$ \\
\hline
\end{tabular}

Fuente: Picado, 2017b y Senado, 1998.

El Cuadro 2 muestra las diferencias marcadas entre ambos enfoques. Se resalta el hecho de que la PS es un modelo social, que permite y propicia la participación de 
los actores sociales, con la idea firme de que sean estos sujetos quienes generen y fomenten los factores protectores de la salud, de forma tal que se propicie, al respecto, un cambio significativo en la cultura de la sociedad en general. Sobre la misma línea, la identificación de los factores protectores de la salud y condiciones favorables en las personas de manera individual, colectiva, comunidades, en lo ambiental, social, económico para gerenciar desde una perspectiva institucional, sectorial e intersectorial procesos de planificación orientados a desarrollar y fortalecer esos aspectos positivos, a fin de mantenerlos y fortalecerlos. Contreras (2013), visualiza lo positivo, hasta ahora ausente en políticas, diagnósticos, planificación, planes, programas y proyectos. De igual forma, propicia el diseño de estrategias dirigidas a establecer criterios de funcionabilidad en la evaluación del impacto de las acciones. En este marco es donde destaca y adquiere relevancia el escenario de la salud.

Por el contrario, la prevención de la enfermedad, si bien es importante, sus actuaciones son limitadas, pues actúa de manera reactiva sobre los factores y grupos de riesgo, por lo que su acción es focalizada, excluyente en tanto deja de lado a otros grupos poblacionales y sus resultados son muy específicos.

\section{Cuadro 3: Enfoques de la PS y de la prevención de enfermedades}

\begin{tabular}{ll}
\hline $\begin{array}{l}\text { Enfoque de necesidades: Prevención de } \\
\text { enfermedades (PE) }\end{array}$ & Enfoque de derechos humanos: Desde PS (PS) \\
\hline El individuo es receptor pasivo & El individuo es un participante activo \\
\hline $\begin{array}{l}\text { Entraña objetivos, generalmente de } \\
\text { atención parcial }\end{array}$ & Entraña el imperativo de atención universal \\
\hline $\begin{array}{l}\text { Es posible satisfacer necesidades sin } \\
\text { garantía de continuidad }\end{array}$ & Deben respetarse siemprelos DDHH \\
\hline $\begin{array}{l}\text { Puede ordenarse de modo jerárquico } \\
\text { No entraña deberes }\end{array}$ & $\begin{array}{l}\text { No tiene organización jerárquica, todos deben } \\
\text { ser respetados }\end{array}$ \\
\hline $\begin{array}{l}\text { Guarda relación con promesa } \\
\text { Puede cambiar según cultura y } \\
\text { circunstancias }\end{array}$ & Entraña deberes \\
\hline Es posible satisfacer por caridad & Son universales. \\
\hline $\begin{array}{l}\text { A menudo la satisfacción depende de } \\
\text { voluntad politica }\end{array}$ & Es inclusivo \\
\hline Se enfoca en las carencias & Valora las posibilidades humanas con obligación \\
\hline $\begin{array}{l}\text { Sus actuaciones son limitadas en cuanto } \\
\text { a desarrollo humano (DH) }\end{array}$ & Favorece el desarrollo humano (DH) general \\
\hline
\end{tabular}

Fuente: Oficina Regional de Asia Meridional, UNICEF, 1998 (citado por Ministerio de Salud, 2011). 


\section{Conclusión}

Al ser la salud un derecho humano universal, los estudios de los diferentes enfoques permiten avanzar en la integración de estos en las políticas públicas y mejorar, por un lado, el acceso de la población y, por otro, la efectividad de dichas políticas en la calidad de vida de las personas.

Históricamente se han establecido enfoques diversos y realizado esfuerzos en torno a PS. Los enfoques planteados se complementan en cuanto favorecen, mediante espacios y formas comunicacionales, el cambio de paradigma para abordar la salud. Involucran, por parte de las comunidades en sus diversas formas de representación, a las instituciones públicas y privadas, a partir de una visión social y trasformadora que realmente genere las condiciones coadyuvantes del desarrollo humano y la calidad de vida de la población.

Mediante los elementos teórico-conceptuales facilitados en este artículo, se pretende también establecer las diferencias significativas entre la lógica positiva de actuación del enfoque de PS en relación con el de prevención de enfermedades

Así mismo, es de suma relevancia señalar que el Ministerio de Salud marca un hito en la historia desde dos puntos de vista: uno, definir claramente las funciones sustantivas y gerenciales de la función rectora de PS, plasmadas en un modelo donde se reconceptualizan términos básicos, de igual forma facilita herramientas que permiten, a los actores sociales del sector salud, sectores varios y sociedad civil en general, clarificar, comprender y operativizar dicho enfoque de acuerdo con sus respectivas competencias. Desde otro punto de vista, se facilita la conducción, articulación de esfuerzos y recursos de estos; todo ello orientado al análisis del escenario de salud, para definir prioridades, planificar conjuntamente, establecer las estrategias, proponer objetivos de satisfacción desde la nueva lógica de actuación de PS positiva, proactiva, propositiva e innovadora; además, impactar, favorablemente, en las condiciones y determinantes de la calidad de vida, considerando los constantes cambios en los diversos ámbitos de acción social.

De lo expuesto se refuerza el rol significativo de la PS en la dimensión social de los determinantes de la salud de la población, lo cual facilitando una visión integradora esencialmente intersectorial y de participación social.

Finalmente, es pertinente señalar que, debido a que la salud es producto de las actuaciones y vínculos sociales, está en cambio constante, por tanto, las organizaciones institucionales públicas y otras vinculadas al tema en cuestión deben también establecer momentos permanentes de revisión e investigación que 
permitan realizar, oportunamente, los ajustes y actualizaciones teórico-conceptual, a normativas metodológicas y operativas que se requieran, para que PS logre resultados e impacte de manera efectiva y positiva en las condiciones y calidad de vida de la población, según características territoriales.

\section{Referencias}

Carmona, L., Rozo, C., Mogollón, A. (2005). La salud y la PS: Una aproximación a su desarrollo histórico y social. Revista Ciencias de la Salud, 3(11), 62-77. Recuperado de https://revistas. urosario.edu.co/index.php/revsalud/article/viewFile/578/497

Cáez, G., Casas, N. (2007). Formar en un estilo de vida saludable: Otro reto para la ingeniería y la industria. Educación y Educadores, 10(2), 103-117. Recuperado de https://www.redalyc. org/pdf/834/83410209.pdf

Contreras, E. (2013). El concepto de estrategia como fundamento de la planeación estratégica. Pensamiento $\mathcal{E}$ Gestión, 35, 152-181. Recuperado de http://www.redalyc.org/ pdf/646/64629832007.pdf

Eslava-Castaneda, J. (2002). Promoción y prevención en el sistema de salud en Colombia. Revista de Salud Pública, 4(1), 1-12. Recuperado de http://www.scielo.org.co/pdf/rsap/v4n1/ v4n1a01.pdf

Eslava-Castaneda, J. (2006). Repensando la PS. Revista de Salud Pública, 8, 106-115. Recuperado de http://www.scielo.org.co/scielo.php?script=sci_arttext\&pid=S0124-00642006000500009

Loreño, E., Moreno, M. (2017). Aportes de la comunicación en los procesos de desarrollo social impulsados por la comisión de justicia social de la provincia de Santa, durante los meses de febrero a septiembre de 2016 (Tesis inédita). Perú: Universidad Nacional del Santa. Recuperado de http:// repositorio.uns.edu.pe/bitstream/handle/UNS/2806/43454.pdf?sequence $=1$ \& isAllowed=y

Maldonado-Vicente, G. (2013). Influencia del coaching con programación neurolingüistica en el desarrollo de competencias en los directivos. San Martín Emprendedor, 4(2), 68-79. Recuperado de http://www.sme.usmp.edu.pe/index.php/sme/article/view/37/79

Maya, L. (2001). Los estilos de vida saludables: Componente de la calidad de vida. San José, Costa Rica: Fundación Colombiana de Tiempo Libre y Recreación Fundación Latinoamericana de Tiempo Libre y Recreación - FUNLIBRE Costa Rica. Recuperado de http://www.funlibre. org/documentos/lemaya1.htm

Ministerio de Salud. (2011). Modelo conceptual y estratégico de la Rectoría de la Producción Social de la Salud. San José, Costa Rica: Dirección General de la Salud. Recuperado de https:/ www.ministeriodesalud.go.cr/index.php/biblioteca-de-archivos/sobre-el-ministerio/modelo-conceptual-y-estrategico/310-modelo-conceptual-y-estrategico-de-la-rectoria-de-la-produccion-social-de-la-salud/ file 
Ministerio de Salud. (2017). Modelo de Rectoría en PS. San José, Costa Rica: Ministerio de Salud. Recuperado de https:/ / www.ministeriodesalud.go.cr/index.php/biblioteca-de-archivos/sobre-el-ministerio/modelo-conceptual-y-estrategico/3742-modelo-de-rectoria-en-promicion-de-la-salud/ file

OMS (1986). Carta de Ottawa para la PS, Primera Conferencia Internacional sobre la PS: Hacia un nuevo concepto de la salud. Toronto, Canadá: Organización Mundial de la Salud. Recuperado de: https://www.paho.org/hq/dmdocuments/2013/Carta-de-ottawa-para-la-apromocion-dela-salud-1986-SP.pdf

Pérez, T., Martínez, E., Rodríguez, L. (2003). Gerencia social integral e incluyente En. Gerencia del desarrollo social: Modelos de acción social. Colombia: Departamento de Investigaciones de la Unidad de Formación Avanzada de la Universidad de la Salle.

Picado, L. (2014). Resultados del estudio de percepciones de actores sociales sobre salud, promoción de la salud, participación social, y de planes municipales región central de occidente, Costa Rica - 2011. Revista Universidad y Salud, 16(2), 150 - 166. Recuperado de http:// www.scielo.org.co/pdf/reus/v16n2/v16n2a03.pdf

Picado, L. (2017 a). Gerencia social en el marco del desarrollo humano: Dos referentes para la implementación del modelo de reorganización y fortalecimiento de la función rectora de PS. San José, Costa Rica: Dirección Regional de Rectoría de la Salud, Ministerio de Salud.

Picado, L. (2017 b). Referentes teórico-conceptuales sobre proceso salud-enfermedad y enfoques de PS; un nuevo paradigma para abordar la salud desde una visión positiva, proactiva, propositiva e innovadora. San José, Costa Rica: Dirección Regional de Rectoría de la Salud, Ministerio de Salud.

Picado, L. y Quesada, A. (2014). Educación continua en PS, desde una acción interinstitucional. Diálogos Revista Electrónica de Historia, 15, 125-143. Recuperado de https:/ / revistas.ucr.ac.cr/ index.php/dialogos/article/view/16305/15807 